\title{
The Photomorphogenic Signal: An Essential Component of Photoautotrophic Life
}

\author{
Sabrina Iñigo, Mariana R. Barber, Maximiliano Sánchez-Lamas, \\ Francisco M. Iglesias and Pablo D. Cerdán \\ Fundación Instituto Leloir, IIBBA-CONICET and FCEN-UBA
}

Argentina

\section{Introduction}

In a thermosolar plant, the engineers locate movable mirrors that concentrate solar radiation. These plants are designed to maximize energy capture. In green plants, their morphology changes to maximize energy capture as well, but also to avoid light in excess, which can damage plant tissues. Contrary to mirrors in thermosolar plants, located in desert land and organized in regular arrays, most green plants grow surrounded of vegetation and their own tissues are not regularly spaced, new leaves tend to shade older leaves. Hence, plants need to use light as a source of information in order to properly locate their "sunlight collectors" and be able to efficiently use light as an energy source for photosynthesis.

To monitor environmental light conditions, plants are equipped with several families of photoreceptors: the cryptochromes, the phytochromes and the LOV-domain bearing photoreceptors. While phytochromes perceive light most effectively in the red/far-red region of the spectrum, cryptochromes and LOV photoreceptors detect blue and UV-A light. Downstream these photoreceptors, plants have evolved sophisticated transcriptional networks that mediate metabolic and developmental changes in response to light. These light-regulated processes include seed germination, seedling photomophogenesis, greening, shade avoidance, photoperiodic responses and senescence.

Greening and chloroplast biogenesis are promoted after light exposure. Phytochromes and cryptochromes trigger to initiate this biogenesis, which includes the induction of photosynthesis-related genes at the transcriptional level, the import of nuclear-encoded proteins and the establishment of a thylakoid network fully assembled with photosynthetic electron transport complexes. Furthermore, these photoreceptors affect the synthesis of chlorophyll and other photosynthetic accessory pigments; modifying the photosynthetic apparatus properties as a result of light quality perception. On the other hand, phytochromes are also involved in the induction of Rubisco, a key enzyme of the Calvin Cycle. Light quality plays an important role in modulating the photosynthetic characteristics. It regulates chlorophyll degradation, modulates photosystem stoichiometry and the activity of the ROS scavenging system.

Besides the role in the formation of the photosynthetic apparatus, the photoreceptors play significant roles in establishing how the photosynthetic pigments will be exposed to light to harvest its energy content. Under weak light, chloroplasts move towards light, in a blue 
light dependent way, to optimize the light absorption and photosynthesis. However, under strong light, chloroplasts show an opposite response to avoid photodamage (Kodama et al., 2011). Besides chloroplast movement, photoreceptors modulate plant architecture to maximize the photosynthetic surface exposed to light. When plants perceive the presence of plant neighbours, phytochromes trigger the elongation of the stem and petioles, a series of changes known as Shade Avoidance Syndrome (SAS). The manipulation of phytochrome levels has been used to improve the harvest index of tobacco plants (Robson et al., 1996) by avoiding the diversion of resources to the SAS. However, the phytochromes are still very important to position the leaves in the canopy. Thus, manipulation of phytochrome activity must be precise, if used to improve crop performance (Maddonni et al., 2002).

In this chapter, we focus on the role of the photomorphogenic signal to trigger the synthesis of photosynthetic genes and pigments during the greening process and later on, during photosynthetic plant development, with emphasis on the regulation of gene expression.

\section{Photomorphogenesis}

Plants are sessile organisms, and as such, have evolved a great deal of developmental plasticity to optimally respond to the immediate environment. Light is one of the most important cues for plant growth; plants respond to its intensity, wavelength, direction and periodicity (Franklin \& Quail, 2009). The first physiological consequence of light perception is the reprogramming of the seedling development in a process termed deetiolation, or photomorphogenesis. In darkness, seedlings display a skotomorphogenesis development characterised by the following phenotypes: elongated hypocotyl, closed, pale and unexpanded cotyledons; the apical hook remains closed to protect the apical meristem before emerging from the soil and chlorophyll and anthocyanin biosynthesis do not take place. All these features allow the seedlings to grow through a layer of soil and eventually emerge into the light. Once the seedlings perceive sufficient light, they exhibit a photomorphogenic development. They undergo deetiolation that includes inhibition of hypocotyl elongation, unfolding and greening of cotyledons, opening of the apical hook, chlorophyll and anthocyanin biosynthesis and differentiation of chloroplasts; processes aimed to achieve full autotrophy. This transition from skotomorphogenic to photomorphogenic development is steered by a complex molecular network that includes upstream signalling components (photoreceptors) and intermediate factors transducing the signal to downstream regulators. These downstream components integrate the light signals from the various photoreceptors and bring about changes in metabolism and gene expression that eventually lead to photomorphogenesis (Casal et al., 2003).

\subsection{Photomorphogenic photoreceptors}

Light is directly perceived by protein molecules known as photoreceptors. Photoreceptors are considered as such if upon photon absorption they are able to deliver a signal to downstream components. Because membranes are transparent to light, most photoreceptors are cytoplasmic and water soluble, contrary to other type of receptors whose ligands are not able to move through membranes.

The solar spectrum at Earth's surface extends from UV (about $280 \mathrm{~nm}$ ) through the blue to red/far red (about $750 \mathrm{~nm}$ ). Because the polypeptide backbone and amino acid side chains do not absorb in most of this range, most photoreceptors contain an organic, non-protein component, known as the chromophore. Chromophores can be attached either covalently or 
non-covalently to the apoprotein (Moglich et al., 2010). As explained above, plants possess several classes of photoreceptors whose absorption properties match the spectrum of the incoming light: the red/far-red photoreversible phytochromes, the UV-A/blue-light absorbing cryptochromes, the phototropins, the members of the Zeitlupe family (Moglich et al., 2010) and, more recently, a UV-B specific photoreceptor, UVR8, has been added to the list (Rizzini et al., 2011).

\subsection{Phytochromes}

\subsubsection{Generalities about phytochromes}

Phytochromes are the only red and far-red light photoreceptors in plants (Strasser et al., 2010; Takano et al., 2009) and, together with cryptochromes and phototropins, constitute one of the three mayor regulators of photomorphogenesis (Rockwell et al., 2006). Phytochromes are synthesised in the cytosol as soluble dimers composed of two 125-kDa polypeptides. Each polypeptide folds into two main domains. The amino-terminal domain covalently binds phytochromobilin (PфB), a tetrapyrrole chromophore that confers the spectral properties characteristic of phytochromes. $\mathrm{P} \phi B$ is synthesised from haeme in plastids, haeme oxygenase encoded by $H Y 1$ converts haeme into biliverdin IX $\alpha$, which is reduced to $3 Z-\mathrm{P} \phi B$ by the $\mathrm{P} \phi \mathrm{B}$ synthase (HY2). Then $3 \mathrm{Z}-\mathrm{P} \phi \mathrm{B}$ isomerises to $3 \mathrm{E}-\mathrm{P} \phi \mathrm{B}$ and attaches covalently to phytochrome (Tanaka \& Tanaka, 2007).

The carboxy-terminal part of the phytochrome molecule is involved in dimerisation and transfer of the signal to downstream components (Rockwell et al., 2006). Phytochromes are synthesised in the dark in a biologically inactive red-light absorbing form (known as Pr). Biological activity is acquired upon red-light triggered photoconversion to the far-red light absorbing form (known as Pfr). Photoconversion of Pfr back to Pr is triggered by far-red light. Both reactions are fully reversible, and eventually results in a dynamic photoequilibrium of Pr and Pfr in natural light conditions that depends on the proportion of red to far-red light (Franklin \& Quail, 2009). The conversion is due to a single photochemical isomerisation of the chromophore about a specific double bond between the rings $\mathrm{C}$ and $\mathrm{D}$ of the phytochromobilin (Rockwell et al., 2006). Following conversion, Pfr translocates into the nucleus (Fankhauser \& Chen, 2008).

The phytochromes are encoded by a small gene family in angiosperms. The rice genome, for example, encodes three members, phyA, phyB and phyC, each representing one of the lineages found in plants (Sharrock, 2008). In Arabidopsis, the phytochrome family consists of five members, designated phytochrome A (phyA) to phytochrome E (phyE).

Classical photobiological experiments established three phytochromes modes of acting, the Very Low Fluence Response mode (VLFR), where responses to phytochromes are already saturated at very low fluencies of light, the Low Fluence Response (LFR) showing the classical red and far-red light reversibility and the High Irradiance responses (HIR) that require prolonged exposures to light of relative high intensity (Casal et al., 2003). Now that we know about each phytochrome species, the phytochrome action modes can be explained by the different phytochrome species and different signal transduction pathways. phyA is the most specialized of the phytochromes; it is responsible for the VLFR and the HIR. The extraordinary sensitivity of this photoreceptor to light allows phyA to control germination of buried seeds in the soil and to induce germination when seeds are exposed briefly to light. phyA importance is evident when plants germinate under a dense canopy (Yanovsky, 1995) or for example, when weeds are induced to germinate after soil tillage (Ballaré, 1992). The other phytochromes control the R/FR reversible LFR and the responses to continuous 
red light. phyB is involved in seed germination, deetiolation, stem elongation, the SAS, stomatal development and several other aspects of plant development. phyD and phyE act in SAS by controlling internode elongation and flowering time, and phyE is also involved in far-red HIR- mediated seed germination (Franklin \& Quail, 2009).

Before even knowing of the existence of multiple phytochromes, they were classified in type I, the light-labile pool and type II, the light stable pool. Now we know that type I is represented by phyA and type II by the other phytochromes. As illustrated above, type I and II phytochromes play distinct roles. The rapid proteolytic degradation of phyA is believed to be responsible for the termination of signalling. The light stable phytochromes are not totally resistant to proteolytic degradation (Jang et al., 2010), but dark reversion also emerges as a switch-off mechanism. Dark reversion is a thermal process in which the Pfr form is slowly converted to the Pr form in the dark. Although dark reversion is not yet well characterised, it makes an important contribution to the balance between Pr and Pfr and hence, to determine the output state for a given phytochrome (Rockwell et al., 2006).

\subsubsection{Phytochrome structure and nuclear translocation}

The two phytochrome mayor domains mentioned above are separated by a flexible hinge region. The N-terminal photosensory region $(70 \mathrm{kDa})$ contains an $\mathrm{N}$-terminal extension (ATE) and three conserved subdomains: PAS, GAF and PHY. The ATE is poorly conserved, possibly accounting for some functional differences among phytochromes and it might be implicated in stabilization of the Pfr form of photoreceptors. The GAF domain is associated with the bilin chromophore and possesses bilin lyase activity. The PAS and PHY domains are important for tuning the spectroscopic properties of the bound bilin.

A flexible hinge region separates the $\mathrm{N}$-terminal domains from the $\mathrm{C}$-terminal regulatory region (55 kDa), which is composed of two PAS subdomains, called PAS 1 and PAS 2, and a histidine kinase related domain (HKRD) (Figure 1). The PAS and HKRD domains contribute to the high-affinity subunit-subunit interaction between the phytochrome monomers to form dimers, and both domains are required for the formation of nuclear speckles. Besides, the PAS domains contain the nuclear localization signal (NLS) responsible for the relocalisation to the nucleus after phytochrome photoconversion (Rockwell et al., 2006). Finally, at least one domain must be responsible for the serine/threonine kinase activity that governs phytochrome autophosphorylation and phytochrome-directed phosphorylation of other proteins, such as PHYTOCHROME-INTERACTING FACTOR 3 (PIF3). The functional significance of this kinase activity remains unknown. HKRD domain was initially suggested to be a kinase because of its relatedness to bacterial histidin kinases (Figure 1). However, it was shown that the kinase activity resides in the N-terminal domain (Bae \& Choi, 2008). Further, it was recently shown that a Casein Kinase II is involved in phosphorylating phytochrome interacting factor 1 (PIF1), one or the downstream effectors of phytochrome signalling (see below) (Bu et al., 2011).

\subsection{Cryptochromes}

Cryptochromes are receptors for blue and ultraviolet light. Arabidopsis contains two cryptochromes, cry1 and cry2. They are composed of two domains, an N-terminal photolyase related region (PHR), without photolyase activity, and a C-terminal extension domain (CCT), more variable among family members (Figure 1). The PHR region binds two chromophores, flavin adenine dinucleotide (FAD) and 5,10-methenyltetrahydrofolate 
(MTFG). The CCT domain appears to be important for cryptochrome function, it interacts with downstream effectors and promotes photomorphogenic development in the dark by itself (Li \& Yang, 2007). cry1 and cry2 form homodimers; dimerisation is mediated by the PRH domain and appears to be essential for signalling (Moglich et al., 2010). cry1 and cry2 are predominantly nuclear. However, cry1 is also found in the cytoplasm. They mediate the regulation of gene expression and together are responsible for blue-light dependent changes in gene expression of up to $10-20 \%$ of the Arabidopsis genome (Lin \& Todo, 2005). cry1 and cry2 participate in many blue-light responses including inhibition of hypocotyl elongation, anthocyanin accumulation, regulation of flowering time, stem and internode elongation, blue-light regulated gene expression, and entrainment of circadian rhythms. The function of cry1 and cry2 partially overlap, but differences are evident at different light intensities or at different developmental stages. Under high intensities of blue light, cry2 is rapidly degraded, leaving cry1 as the predominant photoreceptor, so the role of cry2 during seedling deetiolation is more evident under low blue light intensities. In contrast, cry2 role is predominant in the regulation of flowering time (Li \& Yang, 2007).
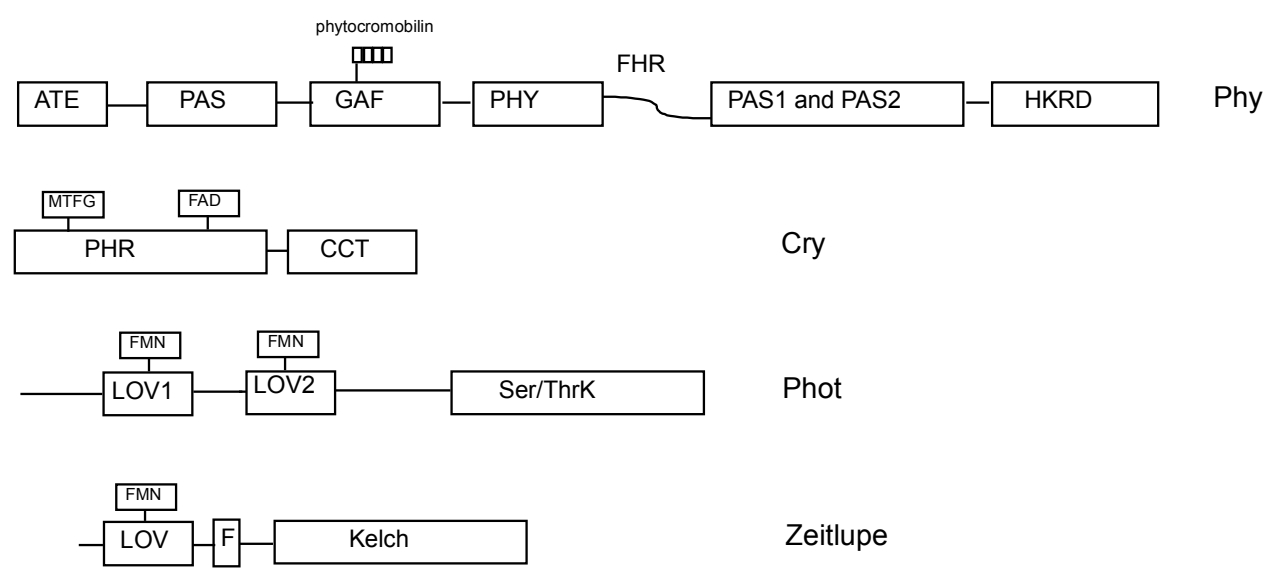

Fig. 1. Schematic representation of domain structure and chromophores of the main photoreceptors: phytochromes (phy), cryptochromes (cry), phototropins (phot) and the zeitlupe family. Domain abbreviation are ATE (N-terminal extension); PAS: domain acronym derived from period clock (PER) protein, aromatic hydrocarbon receptor nuclear translocator (ARNT), and single minded (SIM); GAF: (domain acronym derived from vertebrate cGMP-specific phosphodiesterase, cyanobacterial adenylate cyclase and formate hydrogen lyase transcription activator FhlA); PHY (phytochrome); FHR (flexible hinge region); HKRD (Histidine kinase related domain), PHR (photolyase related region), CCT (Cterminal extension domain), FAD (flavin adenine dinucleotide), MTFG $(5,10$ methenyltetrahydrofolate), LOV (Light-oxygen-voltage domains), Ser/Thrk (serine threonine kinase domain), FMN (Flavin mononucleotide), F (F box), Kelch (Kelch repeat).

\subsection{LOV domain photoreceptors: The phototropins and the ztl family}

The phototropic response of plants has been known at least since Darwin times. The photoreceptors involved were identified after finding mutants impaired in the phototropic response (Huala et al., 1997), and were later named phot1 and phot2. The sequence revealed 
the presence of two domains showing homology to domains that are involved in sensing Light, Oxygen or Voltage, the LOV domains (Figure 1). These domains bind FMN, the chromophore for phototropism. Phototropins were important in the identification of other LOV-domain containing photoreceptors.

The second family of LOV photoreceptors is comprised by Zeitlupe/Adagio/LOV KELCH Protein 1 (ztl/ado/lkp1), fkf1 and lkp2. Contrary to phototropins, the ztl family contains a single LOV domain, an F-box and a C-terminal Kelch domain. F-box proteins play a role in recruiting specific substrates for ubiquitination and protein degradation, whereas the Kelch domain might aid in this function by mediating protein-protein interactions (Moglich et al., 2010). These photoreceptors have important functions in flowering time and circadian clock function, as we will explain in the following sections, mainly by controlling the stability of important clock associated proteins (Harmer, 2009; Mas, 2005).

\subsection{The UV-B specific photoreceptor: The UVR8 protein}

UV-B radiation (280-315 $\mathrm{nm}$ ) is an integral part of the sunlight reaching the surface of the Earth and induces a broad range of physiological responses that are mediated by a recently identified UV-B specific photoreceptor, UVR8 (Rizzini et al., 2011). The most extensively studied examples of photomorphogenic responses are the suppression of hypocotyl extension by low fluences of UV-B and the induction of genes involved in flavonoid biosynthesis (Jenkins, 2009).

UVR8 is a $\beta$-propeller protein with similar sequence to the eukaryotic RCC1, a guanine nucleotide exchange factor (GEF) for the small GTP-binding protein Ran (Gruber et al., 2010). Aromatic amino acids absorb UV-B radiation. Tryptophan, with an absorption maximum in solution at around $280 \mathrm{~nm}$ (which extends to $300 \mathrm{~nm}$ and is likely to be further shifted in a protein environment), is particularly suited as UV-B chromophore. Structure modelling according to structurally related RRC1, identified 14 tryptophans of UVR8 all located at the top of the predicted $\beta$-propeller cluster in the centre of the protein structure. Evidence suggests UV-B perception is based on a tryptophan-based mechanism, an important difference with the other Photoreceptors that bear chromophores suited for visible light perception (Rizzini et al., 2011).

\section{Transducers of light signalling}

\subsection{COP1 is a general repressor of photomorphogenesis}

Most of the photoreceptors mentioned above were identified after genetic screenings in Arabidopsis and led to the isolation of mutants defective in deetiolation. Other type of genetic screenings led to the isolation of mutants with constitutive photomorphogenic phenotypes in the dark (cop) or deetiolated (det). The phenotype of one of such mutants, cop1 (Deng et al., 1992), suggested that it was a negative regulator of photomorphogenesis. COP1 is an essential protein, null alleles are not viable. The overlap between the light-responsive transcriptome and the cop1-responsive transcriptome in dark grown seedlings clearly shows that COP1 is a general repressor of photomorphogenesis (Ma et al., 2002). We now know that COP1 is a single unit E3 ubiquitin ligase, bearing both the substrate and E2 binding motifs. Ubiquitin ligation is the last step in the chain of events that leads to protein ubiquitination that marks proteins for degradation by the $26 \mathrm{~S}$ proteasome. The COP1 protein bears three domains: a RING-finger motif, a coiled-coil domain and seven WD40 repeats. The RING domain is essential to recruit E2s and the other domains to recognize 
substrates. Several of the COP1 substrates have been characterised and they are transcription factors that act positively on photomorphogenesis.

\subsection{COP1 targets positive regulators of photomorphogenesis for degradation 3.2.1 COP1 in phyA signalling}

Genetic and molecular approaches have identified several transcription factors acting positively on photomorphogenesis downstream of photoreceptors. As phyA is the main photoreceptor perceiving continuous far-red light (acting in the HIR mode), mutants with long hypocotyls under far-red light were isolated, leading to phyA signalling components. Two of the genes identified, long after far-red light 1 (laf1) and long hypocotyl in far-red (hfr) encode an R2/R3 MYB and a bHLH transcription factor respectively. Other mutants helped to identify other phyA signalling components; among them, two small plant-specific proteins involved in light-regulated phyA import to the nuclei, FAR-RED ELONGATED HYPOCOTYL1 (FHY1) and its homolog FHY1-LIKE (FHL) (Fankhauser \& Chen, 2008), and two transposase-derived transcription factors, FHY3 and its homolog FAR-RED IMPAIRED RESPONSE1 (FAR1), which are direct activators of FHY1 and FHL transcription, promoting phyA signalling (Lin et al., 2007).

Genetic screenings for enhancers of phyA signalling led to the identification of SUPPRESSOR OF PHYTOCHROME A-105 1 (SPA1), which belongs to a small family of four proteins (SPA1-4). The quadruple mutant defective for the four SPA genes shows a constitutive photomorphogenesis phenotype in the dark, similar to cop1 mutants (Laubinger et al., 2004). SPA proteins and COP1 form complexes and, as mentioned above, show E3 ligase activity (Zhu et al., 2008). This SPA-COP1 complex targets HFR and LAF1 for degradation, explaining part of its negative role in photomorphogenesis (Henriques et al., 2009).

The SPA1-COP1 E3 ligase complex targets other important transcription factors for degradation, like elongated hypocotyl 5 (hy5) and hy5 homolog (hyh), two bZIP transcription factors. These transcription factors promote photomorphogenesis under various wavelengths and will be explained in the following sections.

\subsubsection{COP1 in cryptochrome signalling}

hy5 mutants display a long hypocotyl phenotype under diverse wavelengths of light, suggesting HY5 is a common promotor of photomorphogenesis downstream several photoreceptor signalling pathways. Interestingly, the association between HY5 and COP1 can be deduced from the overlapping set of differentially expressed genes in the respective mutants (Ma et al., 2002). At the biochemical level, it was shown that both HY5 and HYH are targeted for degradation by COP1 (Holm et al., 2002; Osterlund et al., 2000). On the other hand, cryptochromes are known to interact with COP1 through its CCT domain and negatively regulate COP1 activity (Li \& Yang, 2007). However, the precise light-mediated mechanism that controls COP1 activity remained unknown until recently. Three simultaneous publications addressed this issue (Lian et al., 2011; Liu et al., 2011; Zuo et al., 2011). They showed that CRY1 interacts with the SPA proteins in a blue-light dependent manner and inhibit the interaction between COP1 and SPA proteins. This mechanism disrupts the complex E3 ligase activity and avoids HY5 degradation, promoting photomorphogenesis. In the case of CRY2, a similar blue-light dependent interaction with SPA proteins inhibits the activity of the COP1-SPA complex. This inhibition leads to higher levels of CONSTANS, a transcription factor that promotes flowering in long-day conditions. 
These facts also explain some of the differences between the roles of CRY1 and CRY2 in plant development that we mentioned before.

\subsubsection{COP1 in UV-B signalling}

UVR8 forms a dimer but rapidly dissociates as the result of direct perception of UV-B. This is followed by a rapid nuclear accumulation of UVR8 and UVR8 interaction with COP1 that depends on the C-terminal WD40-repeat domain. The UVR8-COP1 interaction mediates the activation of numerous genes, including HY5, inducing photomorphogenic responses (Favory et al., 2009; Jenkins, 2009).

\subsection{The PIF family of bHLH transcription factor represses photomorphogenesis downstream of phytochromes}

The photoconversion of Pr to Pfr with red light leads to conformational changes that unmask the NLS to become accessible for the nuclear-transport machinery and also allow the interacting surfaces for partner proteins. Within the nucleus, phytochromes accumulate in subnuclear foci, the phytochrome Nuclear Bodies (NBs). The identification of HEMERA, a protein involved in the formation of NBs, supports the notion that NBs are the sites of phytochrome-induced protein degradation (Chen et al., 2010). Phytochrome-induced protein degradation is important to control the activity of the Phytochrome interaction factor (PIF) family of bHLH transcription factors. The Pfr form is rapidly translocated into the nucleus, where it interacts with PIFs, more strongly with the Pfr form (Fankhauser \& Chen, 2008). Upon binding Pfr, the PIFs are phosphorylated and degraded. This event initiates a gene expression cascade leading to photomorphogenesis (Bae \& Choi, 2008; Leivar et al., 2009; Shen et al., 2008).

The PIFs belong to a transcription factor superfamily, which forms dimers to target specific DNA sites and are well characterised in nonplant eukaryotes as important regulatory components in diverse biological processes. In Arabidopsis, there are at least 133 bHLH protein-encoding genes. Phylogenetic analysis of the bHLH domain sequences allowed the classification of these genes into 21 subfamilies (Heim et al., 2003; Toledo-Ortiz et al., 2003). The PIFs subfamily, called PHYTOCHROME INTERACTING FACTORs (PIFs) is involved in the repression of seed germination, promotion of seedling skotomorphogenesis and SAS, by regulating the expression of over a thousand genes (Leivar \& Quail, 2011). PIF3 was the first member identified in this subfamily, isolated by a two-hybrid assay as a PHYB interactor ( $\mathrm{Ni}$ et al., 1998). Afterwards, other members of the family were identified by computational analysis (Leivar \& Quail, 2011). Unlike other bHLHs, this subfamily have a characteristic active phytochrome binding motif (APB) in its N-terminal, that make them able to interact with the photoactivated phytochrome (Leivar \& Quail, 2011).

PIFs can form homodimers and heterodimers that bind specifically to the G-box motif CACGTG (Toledo-Ortiz et al., 2003) and, in some cases, HFR1 and other bHLH closely related to PIFs can form non-DNA binding bHLH heterodimers with some PIFs, preventing excessive responses (Hornitschek et al., 2009). In addition, different PIFs are regulated preferentially by different phytochromes (Shen et al., 2008).

As mentioned above, PIF3 is the founding member of this family. PIF3 acts as a negative regulator in both phyA and phyB-mediated seedling deetiolation processes such as hook opening and hypocotyl elongation. Both phyA and phyB bind to PIF3. This interaction leads to the phosphorylation of PIF3, triggering its degradation by the $26 \mathrm{~S}$ proteasome- 
dependent pathway, and thus relieving its negative regulation of photomorphogenesis. phyA is responsible for the rapid degradation of PIF3 in response to far-red light, whereas phyA, phyB and phyD are responsible for PIF3 degradation in response to red light (Bae \& Choi, 2008).

PIF1 (also known as PIL5), PIF4, PIF5 (also known as PIL6) and PIF6 (also known as PIL2) also have important roles in photomorphogenic development. Although they have highly similar sequences, their roles do not overlap completely. For example, PIF1 negatively regulates seed germination by inhibiting gibberelin (GA) biosynthesis and GA signalling, and simultaneously activating abscisic acid biosynthesis. In addition, PIF1 activates the expression of two DELLA genes, which are key negative GA signalling components. Phytochromes promote seed germination by inhibiting PIF1 activity. Conversely, PIF4 and PIF5 have important roles in the regulation of the SAS (Leivar \& Quail, 2011). We will describe the roles of PIFs in chloroplast biogenesis and c chlorophyll synthesis in a following section.

\section{The role of the circadian clock in photomorphogenic development}

The circadian clocks are endogenous mechanisms that allow organisms to time their physiological changes to day/night cycles. These mechanisms are present in a wide range of organisms, from cyanobacteria to mammals. Circadian clocks generate rhythms with a $\sim 24$ hr period, which include changes in gene expression or protein activity. They regulate diverse aspects of plant growth and development, such as the movement of leaves and flowers, the production of volatiles, the stomatal opening, the hypocotyl expansion, the photosynthetic activity and the photoperiodic control of flowering, allowing plants to anticipate daily environmental changes and to synchronise their endogenous physiological processes to external environmental cues. Circadian rhythms persist with a period close to 24 hours after an organism is transferred from an environment that varies according to the time of the day (entraining condition) to an unchanging condition (free-running condition) (Harmer, 2009).

In a simple way, the circadian system can be divided into three main components: the input pathways, involved in the perception and transmission of environmental signals to synchronise the central oscillator that generates and maintains rhythmicity through multiple output pathways, connecting the oscillator to physiology and metabolism. However, this is an oversimplified model of the clock. The circadian system has to be considered as a complex network. The central clock is composed of multiple interlocked feedback loops, where clock outputs may be regulated directly by clock input signalling pathways and can also feedback to clock components and input signalling pathways. Clock genes have multiple functions, they can act within the central oscillator and in clock input and output signalling pathways (Mas, 2005). A key observation is that circadian clock mutants show defective developmental responses to red light (Harmer, 2009), but the endogenous clock oscillates in the absence of phyA phyB cry1 and cry2 (Yanovsky et al., 2000) or in a mutant devoid of all phytochromes (Strasser et al., 2010). These observations imply that the photoreceptors modulate the clock but they are not themselves part of the central oscillator.

\subsection{Molecular basis of the circadian clock}

In Arabidopsis thaliana, the current model for the circadian oscillator is composed of several interlocking positive and negative feedback loops. The first loop that was identified involves 
the Myb-related transcription factors CIRCADIAN CLOCK ASSOCIATED 1 (CCA1) and LATE ELONGATED HYPOCOTYL (LHY) and the pseudo-response regulator TIMING OF CAB EXPRESSION 1 (TOC1/PRR1) (Loop 1, figure 2). CCA1 and LHY proteins have partially redundant functions, bind directly to the TOC1 promoter and inhibit its expression during the day (Alabadi et al., 2001). In turn, TOC1 promotes the expression of CCA1 and LHY indirectly via a hypothetical component $X$ in the morning. The mechanism by which TOC1 induces CCA1 and LHY1 is not completely understood, but it includes CCA1 HIKING EXPEDITION (CHE), a TCP type transcription factor, which associates with TOC1 to regulate CCA1 (Pruneda-Paz et al., 2009). Eventually, CHE also forms an additional loop with CCA1 (Imaizumi, 2010).

Mathematical modelling suggests that an evening-phased negative loop is coupled to the first loop, with an unknown component $Y$ that positively regulates TOC1 whereas $Y$ is negatively regulated by TOC1, CCA1 and LHY (Locke et al., 2005) (Loop 2, figure 2). It was suggested that a portion of $\mathrm{Y}$ activity is provided by the protein GIGANTEA (GI) (Locke $e t$ al., 2005), but this is still unclear (Ito et al., 2009; Martin-Tryon et al., 2007).

The Arabidopsis genome contains four genes encoding proteins with similarity to TOC1: PSEUDORESPONSE REGULATOR (PRR), PRR3, 5, 7 and 9. All these PRR genes play a role in the circadian system, although the effect of single mutations is subtle. Multiple mutants generally have stronger phenotypes, for example the triple prr5 prr7 prr9 mutants are essentially arrhythmic (Nakamichi et al., 2005a; Nakamichi et al., 2005b). Experimental and modelling studies suggest that morning expression of CCA1 and LHY activates the transcription of PRR7 and PRR9 (Farre et al., 2005; Nakamichi et al., 2005b; Zeilinger et al., 2006). This loop is called morning loop (Loop 3, figure 2) and is closed when PRR7 and PRR9 feedback to inhibit CCA1 and $L H Y$ expression. Together the three interlinked feedback loops form an important part of the clock regulatory mechanism and enhance the robustness of the network against environmental perturbations (Harmer, 2009).

Other components that function within or close to the circadian oscillator have recently been identified: FIONA 1, TIME FOR COFFEE, LIGHT REGULATED WD-1 (LWD1) and LWD2 (Ding et al., 2007; Kim et al., 2008; Wu et al., 2008). However, it is not known whether these clock proteins are part of pre-existing loops or constitute unidentified regulatory loops. It has been recently reported that LWD1/2 regulate the expression of multiple oscillator genes and attenuate light signals to adjust period length. Further, it was also proposed that LWD1 and PRR9 form a positive feedback loop within the central oscillator which is also involved in regulating the light input pathway (Wang et al., 2011) (Figure 2). These results underscore the difficulties in dissecting which signalling events are part of the circadian oscillator and which ones are input pathways.

\subsection{Light signalling input to the circadian clock}

Several different photoreceptors mediate light input to the clock, including the phytochromes and the cryptochromes (Somers et al., 1998; Devlin \& Kay, 2000; Yanovsky et al., 2001). However, the molecular mechanisms are only partially known. The ztl family of photoreceptors interacts with clock components and regulates their turnover; hence they are potentially part of input mechanisms. ztl interacts with TOC1 and PRR5, leading to their degradation via the proteasome pathway in the dark (Kiba et al., 2007; Mas et al., 2003). The TOC1-ztl interaction does not depend on light, but an interaction between ztl and GI is bluelight dependent, stabilizes both ztl and GI, and contributes to the robust rhythms of TOC1 
(Kim et al., 2007), contributing to a faster degradation of ztl, GI, TOC1 and PRR5 in darkness than in light (Loop 4, figure 2) (Kiba et al., 2007; Kim et al., 2007; Mas et al., 2003). Within this loop 4, TOC1 binds to PRR3, interfering with TOC1 binding to ztl (Para et al., 2007). Thus, PRR3 seems to stabilize TOC1 avoiding its recruitment to the SCF complex and its degradation by the proteasome (Loop 4, figure 2).

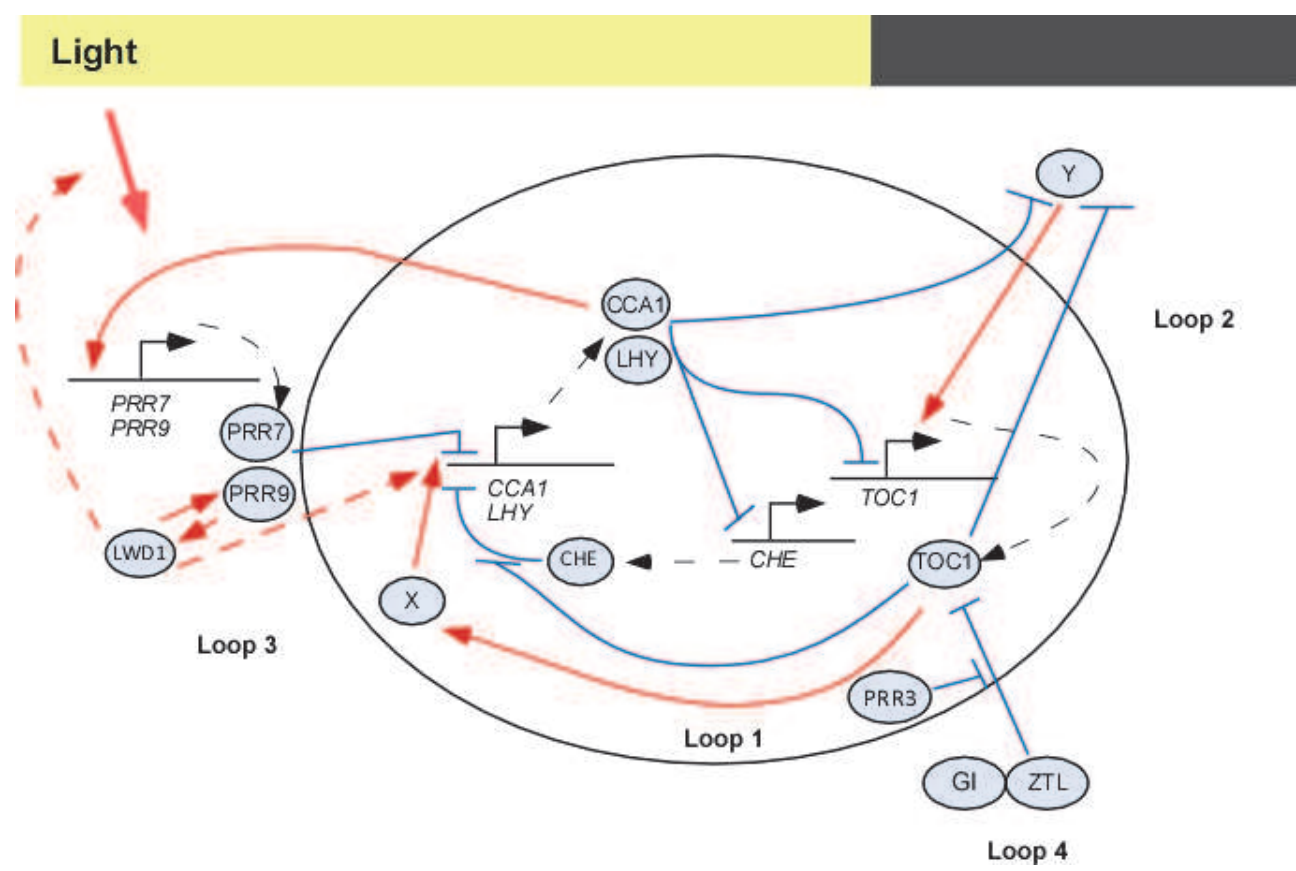

Fig. 2. A model for the Arabidopsis clock. The circadian clock is composed of several interlocking positive and negative loops.

The other members of the ztl family, fkf1 and lkp2, were also studied. Mutant combinations showed that $\mathrm{fkf1}$ and $\mathrm{lkp} 2$ play similar roles to $\mathrm{ztl}$ in the circadian clock when ztl is absent, and that both of them interact with TOC1 and PRR5. These results indicate that ztl, fkf1 and $1 \mathrm{kp} 2$ regulate TOC1 and PRR5 degradation and are important to determine the period of circadian oscillation (Baudry et al., 2010).

Cryptochromes also signal to the circadian clock. However, the mechanisms are still unclear. One possibility is through the regulation of COP1 activity; COP1 directly interacts with ELF3 and with GI to promote GI degradation by the proteasome. This could be a mechanism by which cryptochromes regulate the activity of GI, a protein closely associated with circadian clock function (Yu et al., 2008).

As mentioned above, one interesting aspect of phytochrome and circadian clock is that mutants affected in the clock are also affected in phytochrome responses (Ito et al., 2007). However, how phytochromes contribute to the entrainment of the clock is still unclear. It was previously suggested that PIF3 could directly induce CCA1 and LHY mRNA expression (Martinez-Garcia et al., 2000). Later, it was shown that TOC1 interacts with PIF3 and PIL1 
(Yamashino et al., 2003). However, thorough analysis of PIF3 function has led to the conclusion that it does not play a significant role in controlling light input to the circadian clock (Viczian et al., 2005).

Indeed, there is circumstantial evidence of phytochromes regulating CCA1 and LHY. Both genes are rapidly induced in a TOC1 dependent manner upon transfer of dark grown seedlings to red light. This induction requires EARLY FLOWERING 4 (ELF4), which forms with CCA1 and LHY a negative feedback loop in an analogous manner to TOC1 (Kikis et al., 2005) and ELF4 is itself a direct target of FHY3, FAR1 and HY5 (Li et al., 2011). ELF3, is also necessary for light-induced expression of CCA1 and LHY and this event seems to occur indirectly, through a direct repression of PRR9 by physically interacting with its promoter (Dixon et al., 2011).

\section{Downstream targets of light and clock signalling}

\subsection{The impact of the circadian clock in the expression of photosynthesis related genes}

As presented above, the interconnections between the clock and light signalling are extremely complex. The regulation of outputs is not an exception. One unbiased measure of the impact of the circadian clock on plant development is the finding that at least one third of the Arabidopsis genome is circadian regulated (Covington et al., 2008). The genes involved in photosynthesis are an important target group of the circadian clock, and tend to be expressed at the middle of the subjective day, together with genes involved in the phenylpropanoid pathway (Edwards et al., 2006). In another global analysis it was shown that PRR5, PRR7 and PRR9 are negative regulators of the chlorophyll and carotenoid biosynthetic pathways (Fukushima et al., 2009).

Despite what we know of the clock impact on photosynthetic gene expression, the mechanisms are still poorly understood. One such mechanism may involve CCA1. CCA1 was originally identified by its binding to an AA(CA)AATCT motif in the $l h c b 1^{*} 3$ promoter, and also shown to be required for phytochrome responsivity (Wang et al., 1997). Hence, CCA1 can represent one of the mechanisms by which the clock regulates photosynthetic gene expression. Nevertheless, the reality is more complex. CCA1 binding site is similar to the Evening Element (AAAATATCT) found in promoters of clock regulated genes that peak toward the end of the subjective day (Harmer et al., 2000), including TOC1, which is repressed by CCA1 (Alabadi et al., 2001). However, $l h c b 1^{*} 3$ expression peaks earlier and is promoted by CCA1 (Wang et al., 1997). These apparent contradictions can be reconciled by the finding that CCA1 effects depend on the context, showing also another level of complexity (Harmer \& Kay, 2005).

\subsection{Global expression analysis identifies the targets of photomorphogenesis master regulators}

HY5, the bZIP targeted by COP1 for degradation, is necessary for responses to a broad spectrum of wavelengths of light and, as explained above, acts as a positive regulator in photomorphogenesis. Arabidopsis plants defective in HY5 show aberrant light mediated phenotypes, including an elongated hypocotyl, reduced chlorophyll/anthocyanin accumulation and reduced chloroplast development in greening hypocotyls (Lee et al., 2007). HY5 regulates the transcription of multiple genes in response to light signals through binding to G-box elements in their promoters such as RBCS1A or CHS1 genes. 
Genome-wide CHIP-chip analysis was used to identify HY5 binding regions and to compare this information to HY5-global expression data. This approach allowed the identification of more that 1100 direct targets where HY5 can either activate or repress transcription. However, not all the targets were light responsive genes, suggesting that HY5 must act in concert with other factors to confer light responsiveness (Zhang et al., 2011).

\subsection{The dissection of single light responsive promoters reveals another layer of complexity}

Most of the photoreceptors, signalling components and transcription factors mentioned above were identified using genetic approaches, after Arabidopsis was established as the model plant. Another strategy to understand light signalling and photosynthetic gene expression has been underway since late mid 80s, after the first transgenic plants became available. This strategy was simple, the generation of transgenic plants bearing promoter:reporter gene fusions. With this approach, light responsive promoters were the subject of extensive research with the aim of finding the light responsive elements (LREs) and their cognate binding factors. The genes encoding the small subunits of the Rubisco (RbcS) and the light-harvesting chlorophyll a/b-binding proteins (Lhc; previously known as Cab), were considered a paradigm of light-regulated gene expression (Akhilesh \& Gaur, 2003).

Several LREs were described, as GT-1-Boxes (core sequence GGTTAA), I-Boxes (GATAA), G-Boxes (CACGTG), H-Boxes (CCTACC), AT-rich sequences (consensus AATATTTTTATT) (Akhilesh \& Gaur, 2003). Using complementary approaches as Gel Shift analysis and DNA footprinting, some of the cognate binding factors were identified. However, three difficulties hampered this approaches. First, the LREs identified were not always enough to sustain light regulation. Hence, it was proposed that combinations of different motifs but not multimerisation of single motifs could function as LREs, confirming the complex nature of these regulatory elements (Chattopadhyay et al., 1998; Puente et al., 1996). Second, when the cognate transcription factors were studied in Arabidopsis with available mutants, a direct role in light signal was not evident. This can be illustrated by the GT-element binding factors, a small family of plant trihelix DNAbinding proteins comprising Arabidopsis GT2 (AT1G76890), DF1L (AT1G76880), PTL (At5g03680), GT-2-LIKE1 (GTL1, AT1G33240), GT2L (At5g28300), EDA31 (AT3G10000) and GTL1L (AT5G47660). Some of these transcription factors have roles in the fusion of the polar nuclei, in the development of the embryo sac or even perianth development (Brewer et al., 2004; Pagnussat et al., 2005), but were not involved in responses to light. The third difficulty was the apparent "redundancy" of LREs in single promoters. This redundancy could be just the consequence of a single promoter responding to several different light inputs, as will be explained below.

In a few examples, thorough analysis of promoter sequences, combined with genetic approaches significantly advanced our understanding of light-regulated transcription, but also revealed the complex nature underneath this process. The Arabidopsis Lhcb1*1 (Cab 2) promoter fused to luciferase reporters has been extensively used as a marker for light and circadian expression. Genetic screenings using this construct led to the isolation of toc1 mutants (Strayer et al., 2000). Promoter analysis of $L h c b 1^{*} 1$ allowed the identification of a 78 bp fragment that was sufficient to confer phytochrome and circadian regulation to a minimal promoter (Anderson et al., 1994). Further analysis of this promoter allowed the 
identification of HY5, CCA1 and a DET1 responsive elements (Maxwell et al., 2003). Similarly, it has been shown that HY5 binds to the Lhcb1*3 promoter and physically interacts with CCA1 to synergistically regulate expression (Andronis et al., 2008).

Another promoter analysed in more detail was the tobacco $L h c b 1^{*} 2$. First, a 146 bp promoter fragment sufficed to confer VLFR (mediated by phyA), LFR (mediated by phyB) and HIR (mediated by phyA) to a minimal promoter (Cerdan et al., 1997). Then, the motifs for VLFR and LFR were dissected from the HIR responsive motifs (Cerdan et al., 2000) and finally, the TGGA motif was shown to bind Bell-like homeodomain 1 (BLH1) as part of the phyA mediated HIR (Staneloni et al., 2009). This promoter is an example of how several different photoreceptors can regulate a single gene and integrate their signalling pathways at the promoter level; at least four different photoreceptors were shown to regulate this single promoter (Casal et al., 1998; Cerdan et al., 1999; Mazzella et al., 2001) .

\section{Light promotes chloroplast development}

Proplastids are found in the embryo; they are undifferentiated plastids that are converted to other kind of plastids like chromoplasts, amyloplasts, chloroplasts and etioplasts. During skotomorphogenic development, proplastids turn into etioplasts, the chloroplast precursors. Etioplasts contain the prolamellar body, a structure rich in protochlorophyllide, the chlorophyll precursor, and the enzyme protochlorophyllide oxidoreductase (POR). During the development of etioplasts into chloroplasts, the POR is directly activated by light to convert protochlorophyllide into divinyl-chlorophyllide $a$, which is chlorophyll $a$ and $b$ precursor (Tanaka \& Tanaka, 2007). This light-dependent step can be promoted by red-light in Arabidopsis, even in the absence of phytochromes (Strasser et al., 2010). However, other events that occur during chloroplast biogenesis require the signals transduced by photoreceptors. These signals ensure proper coordination of synthesis and import of LHCB proteins, which are essential for the assembly of the photosynthetic complexes. These events are also coordinated with the synthesis of carotenoids, which are necessary for photoprotection (Cazzonelli \& Pogson, 2010).

Phytochromes, through the action of PIFs, regulate the transition from amiloplasts to etioplasts and to chloroplasts. For example, the PIFs inhibit the conversion of endodermal amyloplasts to etioplasts, whereas the phytochromes antagonise this inhibition, promoting the formation of chloroplasts (Figure 3) (Kim et al., 2011).

\subsection{Chlorophyll biosynthesis is regulated by light}

Chlorophyll biosynthesis and the synthesis of other components of the photosystems are tightly regulated by light and the circadian clock. This coordination is necessary because when the chlorophyll synthesis exceeds the accumulation of chlorophyll-binding apoproteins, reactive oxygen species are generated, ultimately leading to cell death. However, when the chlorophyll synthesis is not enough, the amount of fully functional chlorophyll-binding proteins is not sufficient to gain optimal photosynthetic activity. Another example highlighting the importance of proper coordination is that PIF deficient plants accumulate protochlorophyllide in the dark during skotomorphogenic development, but this accumulation leads to bleaching upon exposure to light (Stephenson et al., 2009).

Plants have four classes of tetrapyrroles: chlorophyll, phytochromobilin, haeme and siroheme, all derived from the same biosynthetic pathway. The flow of the tetrapyrrole pathway is strictly regulated, keeping at low levels the potentially toxic intermediates 
(Tanaka \& Tanaka, 2007). Phytochrome and cryptochrome mutants contain lower levels of chlorophyll (Strasser et al., 2010) stressing out the importance of the photomorphogenic signal for proper assembly of the photosynthetic machinery. In the next paragraphs we review how light signalling pathways regulate chlorophyll biosynthesis (Figure 4).

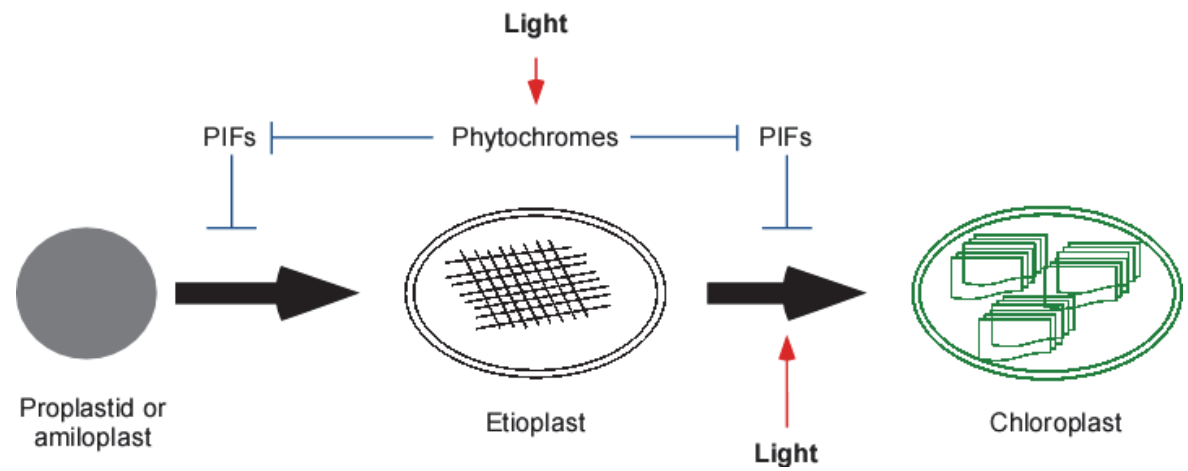

Fig. 3. Light interactions in plastid development. Phytochrome and PIFs roles during the transition from proplastid or amyloplast to chloroplast.

Chlorophyll synthesis occurs in plastids; in the first step glutamate is activated to GlutamyltRNA by the Glutamyl-tRNA synthetase, a step shared with plastid protein synthesis. The following step, the reduction of the Glutamyl-tRNA to produce glutamate-1-semialdehyde is subjected to tight regulation (Figure 4). In Arabidopsis, the Glutamyl-tRNA reductases are encoded by a little family of nuclear genes called HEMA. Of this family, the expression of HEMA1 correlates with the expression of Lhcb1 genes, which encode light-harvesting proteins of the photosystem II; in some way the expression of HEMA1 reflects the demand of chlorophyll synthesis. On the other hand, HEMA2 is not light regulated (Matsumoto et al., 2004; McCormac et al., 2001; McCormac \& Terry, 2002a; McCormac \& Terry, 2002b).

Glutamyl-tRNA reductase activity is regulated by negative feedback loops; the accumulation of Haeme, Mg-Protoporphyrin IX or Divinyl protochlorofilide a antagonise Glutamyl-tRNA reductase activity (Srivastava et al., 2005). At the transcriptional level, HEMA1 expression is induced by red and far-red light, implicating at least phyA and phyB, and blue light perceived by cry1 (McCormac et al., 2001; McCormac \& Terry, 2002a). pif1 and pif3 mutants contain higher levels of HEMA1 mRNA, higher levels of protochlorophyllide and partially developed chloroplasts in the dark, a phenotype observed in cop mutants. The effects of pif1 and pif3 mutations are essentially additive, suggesting a model where phytochromes promote chloroplast biogenesis by antagonizing the activity of at least PIF1 and PIF3. As PIF1 and PIF3 are regulated by the circadian clock, but do not seem to affect central clock components (TOC1, CCA1, LHY), these PIFs seem to integrate chloroplast biogenesis with circadian and light signalling (Stephenson et al., 2009).

The expression of photosynthetic nuclear genes is repressed by plastid signals if chloroplast biogenesis is blocked (retrograde signalling). This finding led to the isolation of mutants that disrupt chloroplast to nucleus communication, the genomes uncoupled mutants (gun) (Nott et al., 2006). These mutants show high levels of lhcb1 mRNA in the presence of norfluorzazon and were named gun1 to gun5. gun 2 and gun3 are allelic to hy1 and hy2 and disrupt phytochromobilin synthesis, leading to haeme accumulation and feedback 
inhibition of Glutamyl-tRNA reductase (Nott et al., 2006). The product of the GUN4 gene, a $22 \mathrm{kD}$ protein localized to Chloroplasts, promotes Magnesium chelatase $(\mathrm{MgCH})$ activity which catalyses the insertion of $\mathrm{Mg} 2+$ into protoporphyrin IX (Tanaka \& Tanaka, 2007). The GUN4 gene is also under circadian clock regulation and is repressed by PIF1 and PIF3 suggesting a similar regulatory mechanism to HEMA1 (Stephenson et al., 2009). The expression of GUN4 is primarily under the control of phyA and phyB with some input from

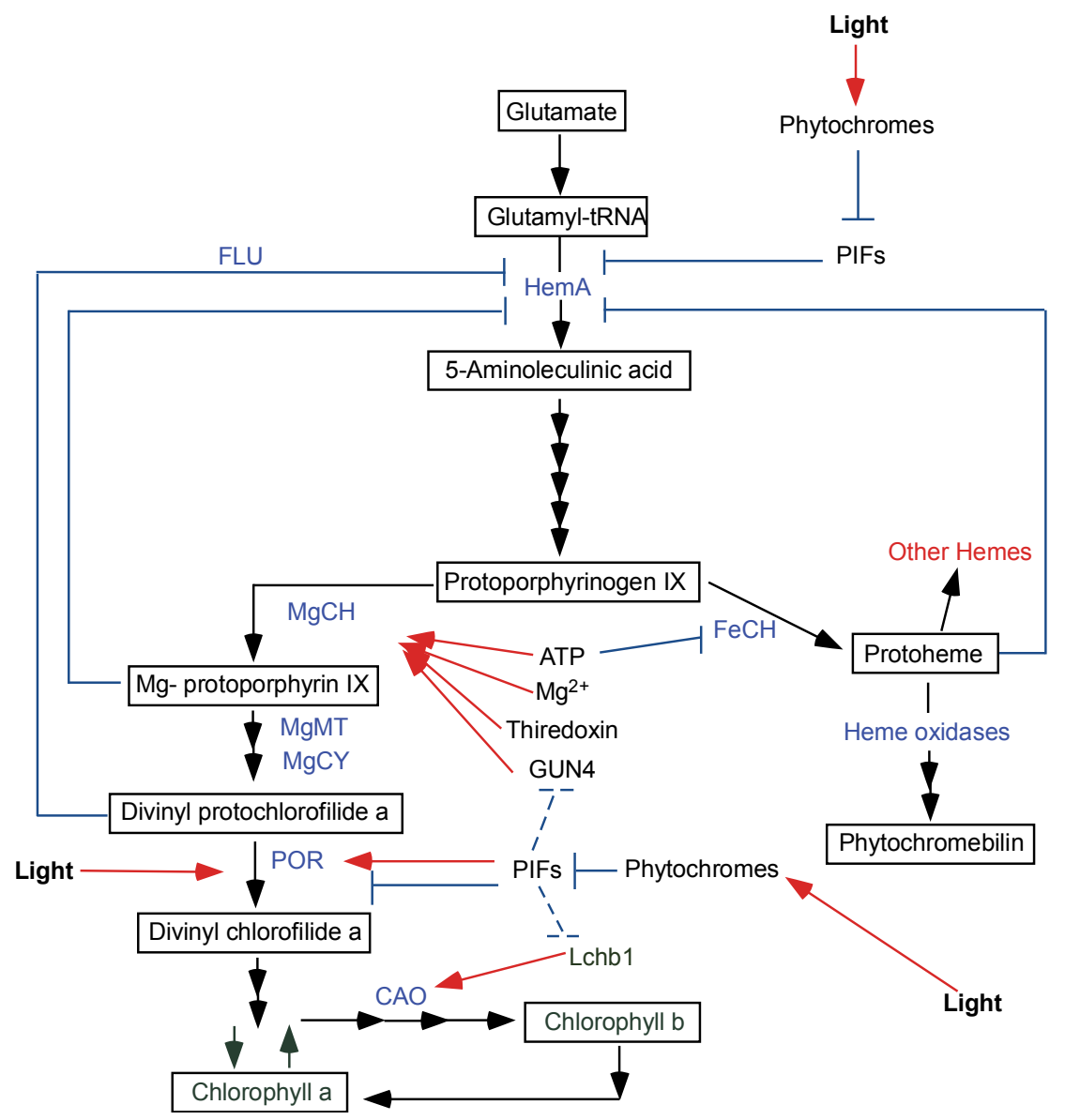

Fig. 4. Simplified chlorophyll biosynthesis pathway and light regulated steps. We emphasise how the light regulate directly the activity of NADPH:protochlorophyllide oxidoreductase (POR); or indirectly, through phytochrome and PIFs the expression of genes encoding the Glutamyl-tRNA reductases (HEMAs), Ferrum chelatase $(\mathrm{FeCH})$, Magnesium chelatase $(\mathrm{MgCH}), \mathrm{NADPH}$ :protochlorophyllide oxidoreductase (POR), Chlorophyllide a oxygenase (CAO), Mg-protoporphyrin IX methyltransferase (MgMT), and Mg-protoporphyrin IX monomethyl estercyclase (MgCy). The ATP/ADP ratio, the $\mathrm{Mg}^{2+}$ concentration and the thioredoxin levels also affect the $\mathrm{MgCH}$ activity, furthermore, these factors are light regulated (Tanaka \& Tanaka, 2007). LHCs attach chlorophyll $a$, and CAO converts the chlorophyll a to $b$ on the LHC apoprotein (Tanaka \& Tanaka, 2007). 
the cryptochromes, establishing GUN4 as a link between the phytochromes and the regulation of $\mathrm{MgCH}$ activity (Stephenson \& Terry, 2008). GUN5 encodes the $\mathrm{H}$ subunit of $\mathrm{MgCH}$, known as CHLH (Nott et al., 2006). The expression of CHLH is regulated at the mRNA level by light/dark cycles and by the circadian clock. Interestingly, this gene is co-regulated with HEMA1, lhcb, Mg-protoporphyrin IX monomethyl estercyclase (MGCy) and the gene encoding the chlorophyll(ide) a oxygenase (CAO) (Matsumoto et al., 2004). On the other hand, GUN1 encodes a pentatricopeptide repeat-containing protein that does not affect chlorophyll synthesis. GUN1 was proposed to generate a signal in chloroplast that represses nuclear photosynthetic gene expression; this repression on $l h c b$ genes seems to be mediated by direct binding of ABI4, an AP2-type transcription factor (Koussevitzky et al., 2007).

Another connection between light signalling and the retrograde signalling was recently established. A sensitive genetic screening for the gun phenotype uncovered new cry1 alleles. These results establish that cry1 is necessary for maximal repression of $l h c b$ genes, when chloroplast biogenesis is blocked (Ruckle et al., 2007).

One of the latest steps in chlorophyll synthesis is the reduction of 3,8-divinyl protochlorophyllide to 3,8-divinyl chlorophyllide. This protochlorophyllide to chlorophyllide conversion is catalysed by the POR enzyme. In angiosperms, POR is light-dependent and it is likely the source of red-light promoted chlorophyll synthesis in the absence of phytochromes (Strasser et al., 2010). Angiosperms carry three POR-encoding genes, PorA, PorB and PorC, which are differentially regulated by both light and developmental stage. PORA expression is high in etiolated seedlings and rapidly becomes undetectable after illumination with FR, a HIR response mediated by phyA, whereas PORB expression persists throughout greening and in adult plants (Runge et al., 1996). PORC is expressed during the adult life and together with PORB is responsible for bulk chlorophyll synthesis in green plants (Paddock et al., 2010). It has been recently shown that PORC expression is directly activated by PIF1 binding to a G-box in PORC promoter, whereas PORA and PORB are also induced by PIF1, presumably in an indirect manner (Moon et al., 2008).

\section{Conclusion}

During the last twenty years, plant biologists have witnessed major advances in our understanding of how plants use light as a source of information. These advances were possible thanks to the adoption of Arabidopsis as a model system. During these twenty years, 13 Arabidopsis photoreceptors were characterised In molecular terms and these findings extended to other species as well. A high number of signal transduction components were also characterised. With the advent of "omics" technologies, the networks that work downstream photoreceptors and their targets started to surface. However, with all these advances, we still do not know in detail how a single light responsive promoter works. How many transcription factors are sitting there? Which are their identities? How do they interact to fine tune expression under the diverse light conditions found in nature? If we multiply these questions by the number of light responsive promoters we can just have a hint of the enormous task ahead.

\section{References}

Akhilesh, K. T. \&Gaur, T. (2003). Light regulation of nuclear photosynthetic genes in higher plants. Critical Reviews in Plant Sciences 22(5): 417-452. 
Alabadi, D., Oyama, T., Yanovsky, M. J., Harmon, F. G., Mas, P. \&Kay, S. A. (2001). Reciprocal regulation between TOC1 and LHY/CCA1 within the Arabidopsis circadian clock. Science 293(5531): 880-883.

Anderson, S. L., Teakle, G. R., Martino-Catt, S. J. \&Kay, S. A. (1994). Circadian clock- and phytochrome-regulated transcription is conferred by a $78 \mathrm{bp}$ cis-acting domain of the Arabidopsis CAB2 promoter. Plant J 6(4): 457-470.

Andronis, C., Barak, S., Knowles, S. M., Sugano, S. \&Tobin, E. M. (2008). The clock protein CCA1 and the bZIP transcription factor HY5 physically interact to regulate gene expression in Arabidopsis. Mol Plant 1(1): 58-67.

Bae, G. \&Choi, G. (2008). Decoding of light signals by plant phytochromes and their interacting proteins. Annu Rev Plant Biol 59: 281-311.

Ballaré, C. L. S., A.L.; Sánchez, R.A.; Radosevich, S. (1992). Photomorphogenic process in the agricultural environment. Photochemistry and Photobiology 56: 12.

Baudry, A., Ito, S., Song, Y. H., Strait, A. A., Kiba, T., Lu, S., Henriques, R., Pruneda-Paz, J. L., Chua, N. H., Tobin, E. M., Kay, S. A. \&Imaizumi, T. (2010). F-box proteins FKF1 and LKP2 act in concert with ZEITLUPE to control Arabidopsis clock progression. Plant Cell 22(3): 606-622.

Brewer, P. B., Howles, P. A., Dorian, K., Griffith, M. E., Ishida, T., Kaplan-Levy, R. N., Kilinc, A. \&Smyth, D. R. (2004). PETAL LOSS, a trihelix transcription factor gene, regulates perianth architecture in the Arabidopsis flower. Development 131(16): 4035-4045.

Bu, Q., Zhu, L., Dennis, M. D., Yu, L., Lu, S. X., Person, M. D., Tobin, E. M., Browning, K. S. \&Huq, E. (2011). Phosphorylation by CK2 enhances the rapid light-induced degradation of phytochrome interacting factor 1 in Arabidopsis. J Biol Chem 286(14): 12066-12074.

Casal, J. J., Cerdan, P. D., Staneloni, R. J. \&Cattaneo, L. (1998). Different phototransduction kinetics of phytochrome A and phytochrome B in Arabidopsis thaliana. Plant Physiol 116(4): 1533-1538.

Casal, J. J., Luccioni, L. G., Oliverio, K. A. \&Boccalandro, H. E. (2003). Light, phytochrome signalling and photomorphogenesis in Arabidopsis. Photochem Photobiol Sci 2(6): 625-636.

Cazzonelli, C. I. \&Pogson, B. J. (2010). Source to sink: regulation of carotenoid biosynthesis in plants. Trends Plant Sci 15(5): 266-274.

Cerdan, P. D., Staneloni, R. J., Casal, J. J. \&Sanchez, R. A. (1997). A 146 bp fragment of the tobacco Lhcb1*2 promoter confers very-low-fluence, low-fluence and highirradiance responses of phytochrome to a minimal CaMV 35S promoter. Plant Mol Biol 33(2): 245-255.

Cerdan, P. D., Staneloni, R. J., Ortega, J., Bunge, M. M., Rodriguez-Batiller, M. J., Sanchez, R. A. \&Casal, J. J. (2000). Sustained but not transient phytochrome A signaling targets a region of an Lhcb1*2 promoter not necessary for phytochrome B action. Plant Cell 12(7): 1203-1211.

Cerdan, P. D., Yanovsky, M. J., Reymundo, F. C., Nagatani, A., Staneloni, R. J., Whitelam, G. C. \&Casal, J. J. (1999). Regulation of phytochrome B signaling by phytochrome A and FHY1 in Arabidopsis thaliana. Plant J 18(5): 499-507.

Covington, M. F., Maloof, J. N., Straume, M., Kay, S. A. \&Harmer, S. L. (2008). Global transcriptome analysis reveals circadian regulation of key pathways in plant growth and development. Genome Biol 9(8): R130.

Chattopadhyay, S., Puente, P., Deng, X. W. \&Wei, N. (1998). Combinatorial interaction of light-responsive elements plays a critical role in determining the response characteristics of light-regulated promoters in Arabidopsis. Plant J 15(1): 69-77. 
Chen, M., Galvao, R. M., Li, M., Burger, B., Bugea, J., Bolado, J. \&Chory, J. (2010). Arabidopsis HEMERA/pTAC12 initiates photomorphogenesis by phytochromes. Cell 141(7): 1230-1240.

Deng, X. W., Matsui, M., Wei, N., Wagner, D., Chu, A. M., Feldmann, K. A. \&Quail, P. H. (1992). COP1, an Arabidopsis regulatory gene, encodes a protein with both a zincbinding motif and a G beta homologous domain. Cell 71(5): 791-801.

Devlin, P. F. \&Kay, S. A. (2000). Cryptochromes are required for phytochrome signaling to the circadian clock but not for rhythmicity. Plant Cell 12(12): 2499-2510.

Ding, Z., Millar, A. J., Davis, A. M. \&Davis, S. J. (2007). TIME FOR COFFEE encodes a nuclear regulator in the Arabidopsis thaliana circadian clock. Plant Cell 19(5): 15221536.

Dixon, L. E., Knox, K., Kozma-Bognar, L., Southern, M. M., Pokhilko, A. \&Millar, A. J. (2011). Temporal repression of core circadian genes is mediated through EARLY FLOWERING 3 in Arabidopsis. Curr Biol 21(2): 120-125.

Edwards, K. D., Anderson, P. E., Hall, A., Salathia, N. S., Locke, J. C., Lynn, J. R., Straume, M., Smith, J. Q. \&Millar, A. J. (2006). FLOWERING LOCUS C mediates natural variation in the high-temperature response of the Arabidopsis circadian clock. Plant Cell 18(3): 639-650.

Fankhauser, C. \&Chen, M. (2008). Transposing phytochrome into the nucleus. Trends Plant Sci 13(11): 596-601.

Farre, E. M., Harmer, S. L., Harmon, F. G., Yanovsky, M. J. \&Kay, S. A. (2005). Overlapping and distinct roles of PRR7 and PRR9 in the Arabidopsis circadian clock. Curr Biol 15(1): 47-54.

Favory, J. J., Stec, A., Gruber, H., Rizzini, L., Oravecz, A., Funk, M., Albert, A., Cloix, C., Jenkins, G. I., Oakeley, E. J., Seidlitz, H. K., Nagy, F. \&Ulm, R. (2009). Interaction of COP1 and UVR8 regulates UV-B-induced photomorphogenesis and stress acclimation in Arabidopsis. Embo J 28(5): 591-601.

Franklin, K. A. \&Quail, P. H. (2009). Phytochrome functions in Arabidopsis development. J Exp Bot 61(1): 11-24.

Fukushima, A., Kusano, M., Nakamichi, N., Kobayashi, M., Hayashi, N., Sakakibara, H., Mizuno, T. \&Saito, K. (2009). Impact of clock-associated Arabidopsis pseudoresponse regulators in metabolic coordination. Proc Natl Acad Sci U S A 106(17): 7251-7256.

Gruber, H., Heijde, M., Heller, W., Albert, A., Seidlitz, H. K. \&Ulm, R. (2010). Negative feedback regulation of UV-B-induced photomorphogenesis and stress acclimation in Arabidopsis. Proc Natl Acad Sci U S A 107(46): 20132-20137.

Harmer, S. L. (2009). The circadian system in higher plants. Annu Rev Plant Biol 60: 357-377.

Harmer, S. L., Hogenesch, J. B., Straume, M., Chang, H. S., Han, B., Zhu, T., Wang, X., Kreps, J. A. \&Kay, S. A. (2000). Orchestrated transcription of key pathways in Arabidopsis by the circadian clock. Science 290(5499): 2110-2113.

Harmer, S. L. \&Kay, S. A. (2005). Positive and negative factors confer phase-specific circadian regulation of transcription in Arabidopsis. Plant Cell 17(7): 1926-1940.

Heim, M. A., Jakoby, M., Werber, M., Martin, C., Weisshaar, B. \&Bailey, P. C. (2003). The basic helix-loop-helix transcription factor family in plants: a genome-wide study of protein structure and functional diversity. Mol Biol Evol 20(5): 735-747.

Henriques, R., Jang, I. C. \&Chua, N. H. (2009). Regulated proteolysis in light-related signaling pathways. Curr Opin Plant Biol 12(1): 49-56. 
Holm, M., Ma, L. G., Qu, L. J. \&Deng, X. W. (2002). Two interacting bZIP proteins are direct targets of COP1-mediated control of light-dependent gene expression in Arabidopsis. Genes Dev 16(10): 1247-1259.

Hornitschek, P., Lorrain, S., Zoete, V., Michielin, O. \&Fankhauser, C. (2009). Inhibition of the shade avoidance response by formation of non-DNA binding bHLH heterodimers. EMBO J 28(24): 3893-3902.

Huala, E., Oeller, P. W., Liscum, E., Han, I. S., Larsen, E. \&Briggs, W. R. (1997). Arabidopsis NPH1: a protein kinase with a putative redox-sensing domain. Science 278(5346): 2120-2123.

Imaizumi, T. (2010). Arabidopsis circadian clock and photoperiodism: time to think about location. Curr Opin Plant Biol 13(1): 83-89.

Ito, S., Kawamura, H., Niwa, Y., Nakamichi, N., Yamashino, T. \&Mizuno, T. (2009). A genetic study of the Arabidopsis circadian clock with reference to the TIMING OF CAB EXPRESSION 1 (TOC1) gene. Plant Cell Physiol 50(2): 290-303.

Ito, S., Nakamichi, N., Kiba, T., Yamashino, T. \&Mizuno, T. (2007). Rhythmic and lightinducible appearance of clock-associated pseudo-response regulator protein PRR9 through programmed degradation in the dark in Arabidopsis thaliana. Plant Cell Physiol 48(11): 1644-1651.

Jang, I. C., Henriques, R., Seo, H. S., Nagatani, A. \&Chua, N. H. (2010). Arabidopsis PHYTOCHROME INTERACTING FACTOR proteins promote phytochrome B polyubiquitination by COP1 E3 ligase in the nucleus. Plant Cell 22(7): 2370-2383.

Jenkins, G. I. (2009). Signal transduction in responses to UV-B radiation. Annu Rev Plant Biol 60: 407-431.

Kiba, T., Henriques, R., Sakakibara, H. \&Chua, N. H. (2007). Targeted degradation of PSEUDO-RESPONSE REGULATOR5 by an SCFZTL complex regulates clock function and photomorphogenesis in Arabidopsis thaliana. Plant Cell 19(8): 25162530.

Kikis, E. A., Khanna, R. \&Quail, P. H. (2005). ELF4 is a phytochrome-regulated component of a negative-feedback loop involving the central oscillator components CCA1 and LHY. Plant J 44(2): 300-313.

Kim, J., Kim, Y., Yeom, M., Kim, J. H. \&Nam, H. G. (2008). FIONA1 is essential for regulating period length in the Arabidopsis circadian clock. Plant Cell 20(2): 307319.

Kim, K., Shin, J., Lee, S. H., Kweon, H. S., Maloof, J. N. \&Choi, G. (2011). Phytochromes inhibit hypocotyl negative gravitropism by regulating the development of endodermal amyloplasts through phytochrome-interacting factors. Proc Natl Acad Sci U S A 108(4): 1729-1734.

Kim, W. Y., Fujiwara, S., Suh, S. S., Kim, J., Kim, Y., Han, L., David, K., Putterill, J., Nam, H. G. \&Somers, D. E. (2007). ZEITLUPE is a circadian photoreceptor stabilized by GIGANTEA in blue light. Nature 449(7160): 356-360.

Kodama, Y., Suetsugu, N. \&Wada, M. (2011). Novel protein-protein interaction family proteins involved in chloroplast movement response. Plant Signal Behav 6(4): 483490.

Koussevitzky, S., Nott, A., Mockler, T. C., Hong, F., Sachetto-Martins, G., Surpin, M., Lim, J., Mittler, R. \&Chory, J. (2007). Signals from chloroplasts converge to regulate nuclear gene expression. Science 316(5825): 715-719.

Laubinger, S., Fittinghoff, K. \&Hoecker, U. (2004). The SPA quartet: a family of WD-repeat proteins with a central role in suppression of photomorphogenesis in arabidopsis. Plant Cell 16(9): 2293-2306. 
Lee, J., He, K., Stolc, V., Lee, H., Figueroa, P., Gao, Y., Tongprasit, W., Zhao, H., Lee, I. \&Deng, X. W. (2007). Analysis of transcription factor HY5 genomic binding sites revealed its hierarchical role in light regulation of development. Plant Cell 19(3): 731-749.

Leivar, P. \&Quail, P. H. (2011). PIFs: pivotal components in a cellular signaling hub. Trends Plant Sci 16(1): 19-28.

Leivar, P., Tepperman, J. M., Monte, E., Calderon, R. H., Liu, T. L. \&Quail, P. H. (2009). Definition of Early Transcriptional Circuitry Involved in Light-Induced Reversal of PIF-Imposed Repression of Photomorphogenesis in Young Arabidopsis Seedlings. Plant Cell.

Li, Q. H. \&Yang, H. Q. (2007). Cryptochrome signaling in plants. Photochem Photobiol 83(1): 94-101.

Li, G., Siddiqui, H., Teng, Y., Lin, R., Wan, X. Y., Li, J., Lau, O. S., Ouyang, X., Dai, M., Wan, J., Devlin, P. F., Deng, X. W. \&Wang, H. (2011). Coordinated transcriptional regulation underlying the circadian clock in Arabidopsis. Nat Cell Biol 13(5): 616622.

Lian, H. L., He, S. B., Zhang, Y. C., Zhu, D. M., Zhang, J. Y., Jia, K. P., Sun, S. X., Li, L. \&Yang, H.Q. (2011). Blue-light-dependent interaction of cryptochrome 1 with SPA1 defines a dynamic signaling mechanism. Genes Dev 25(10): 1023-1028.

Lin, C. \&Todo, T. (2005). The cryptochromes. Genome Biol 6(5): 220.

Lin, R., Ding, L., Casola, C., Ripoll, D. R., Feschotte, C. \&Wang, H. (2007). Transposasederived transcription factors regulate light signaling in Arabidopsis. Science 318(5854): 1302-1305.

Liu, B., Zuo, Z., Liu, H., Liu, X. \&Lin, C. (2011). Arabidopsis cryptochrome1 interacts with SPA1 to suppress COP1 activity in response to blue light. Genes Dev 25(10): 10291034.

Locke, J. C., Southern, M. M., Kozma-Bognar, L., Hibberd, V., Brown, P. E., Turner, M. S. \&Millar, A. J. (2005). Extension of a genetic network model by iterative experimentation and mathematical analysis. Mol Syst Biol 1: 20050013.

Ma, L., Gao, Y., Qu, L., Chen, Z., Li, J., Zhao, H. \&Deng, X. W. (2002). Genomic evidence for COP1 as a repressor of light-regulated gene expression and development in Arabidopsis. Plant Cell 14(10): 2383-2398.

Maddonni, G. A., Otegui, M. E., Andrieu, B., Chelle, M. \&Casal, J. J. (2002). Maize leaves turn away from neighbors. Plant Physiol 130(3): 1181-1189.

Martin-Tryon, E. L., Kreps, J. A. \&Harmer, S. L. (2007). GIGANTEA acts in blue light signaling and has biochemically separable roles in circadian clock and flowering time regulation. Plant Physiol 143(1): 473-486.

Martinez-Garcia, J. F., Huq, E. \&Quail, P. H. (2000). Direct targeting of light signals to a promoter element-bound transcription factor. Science 288(5467): 859-863.

Mas, P. (2005). Circadian clock signaling in Arabidopsis thaliana: from gene expression to physiology and development. Int J Dev Biol 49(5-6): 491-500.

Mas, P., Kim, W. Y., Somers, D. E. \&Kay, S. A. (2003). Targeted degradation of TOC1 by ZTL modulates circadian function in Arabidopsis thaliana. Nature 426(6966): 567-570.

Matsumoto, F., Obayashi, T., Sasaki-Sekimoto, Y., Ohta, H., Takamiya, K. \&Masuda, T. (2004). Gene expression profiling of the tetrapyrrole metabolic pathway in Arabidopsis with a mini-array system. Plant Physiol 135(4): 2379-2391.

Maxwell, B. B., Andersson, C. R., Poole, D. S., Kay, S. A. \&Chory, J. (2003). HY5, Circadian Clock-Associated 1, and a cis-element, DET1 dark response element, mediate DET1 
regulation of chlorophyll a/b-binding protein 2 expression. Plant Physiol 133(4): 1565-1577.

Mazzella, M. A., Cerdan, P. D., Staneloni, R. J. \&Casal, J. J. (2001). Hierarchical coupling of phytochromes and cryptochromes reconciles stability and light modulation of Arabidopsis development. Development 128(12): 2291-2299.

McCormac, A. C., Fischer, A., Kumar, A. M., Soll, D. \&Terry, M. J. (2001). Regulation of HEMA1 expression by phytochrome and a plastid signal during de-etiolation in Arabidopsis thaliana. Plant J 25(5): 549-561.

McCormac, A. C. \&Terry, M. J. (2002a). Light-signalling pathways leading to the coordinated expression of HEMA1 and Lhcb during chloroplast development in Arabidopsis thaliana. Plant J 32(4): 549-559.

McCormac, A. C. \&Terry, M. J. (2002b). Loss of nuclear gene expression during the phytochrome A-mediated far-red block of greening response. Plant Physiol 130(1): 402-414.

Moglich, A., Yang, X., Ayers, R. A. \&Moffat, K. (2010). Structure and function of plant photoreceptors. Annu Rev Plant Biol 61: 21-47.

Moon, J., Zhu, L., Shen, H. \&Huq, E. (2008). PIF1 directly and indirectly regulates chlorophyll biosynthesis to optimize the greening process in Arabidopsis. Proc Natl Acad Sci U S A 105(27): 9433-9438.

Nakamichi, N., Kita, M., Ito, S., Sato, E., Yamashino, T. \&Mizuno, T. (2005a). The Arabidopsis pseudo-response regulators, PRR5 and PRR7, coordinately play essential roles for circadian clock function. Plant Cell Physiol 46(4): 609-619.

Nakamichi, N., Kita, M., Ito, S., Yamashino, T. \&Mizuno, T. (2005b). PSEUDO-RESPONSE REGULATORS, PRR9, PRR7 and PRR5, together play essential roles close to the circadian clock of Arabidopsis thaliana. Plant Cell Physiol 46(5): 686-698.

Ni, M., Tepperman, J. M. \&Quail, P. H. (1998). PIF3, a phytochrome-interacting factor necessary for normal photoinduced signal transduction, is a novel basic helix-loophelix protein. Cell 95(5): 657-667.

Nott, A., Jung, H. S., Koussevitzky, S. \&Chory, J. (2006). Plastid-to-nucleus retrograde signaling. Annu Rev Plant Biol 57: 739-759.

Osterlund, M. T., Hardtke, C. S., Wei, N. \&Deng, X. W. (2000). Targeted destabilization of HY5 during light-regulated development of Arabidopsis. Nature 405(6785): 462-466.

Paddock, T. N., Mason, M. E., Lima, D. F. \&Armstrong, G. A. (2010). Arabidopsis protochlorophyllide oxidoreductase A (PORA) restores bulk chlorophyll synthesis and normal development to a porB porC double mutant. Plant Mol Biol 72(4-5): 445457.

Pagnussat, G. C., Yu, H. J., Ngo, Q. A., Rajani, S., Mayalagu, S., Johnson, C. S., Capron, A., Xie, L. F., Ye, D. \&Sundaresan, V. (2005). Genetic and molecular identification of genes required for female gametophyte development and function in Arabidopsis. Development 132(3): 603-614.

Para, A., Farre, E. M., Imaizumi, T., Pruneda-Paz, J. L., Harmon, F. G. \&Kay, S. A. (2007). PRR3 Is a vascular regulator of TOC1 stability in the Arabidopsis circadian clock. Plant Cell 19(11): 3462-3473.

Pruneda-Paz, J. L., Breton, G., Para, A. \&Kay, S. A. (2009). A functional genomics approach reveals $\mathrm{CHE}$ as a component of the Arabidopsis circadian clock. Science 323(5920): 1481-1485.

Puente, P., Wei, N. \&Deng, X. W. (1996). Combinatorial interplay of promoter elements constitutes the minimal determinants for light and developmental control of gene expression in Arabidopsis. Embo J 15(14): 3732-3743. 
Rizzini, L., Favory, J. J., Cloix, C., Faggionato, D., O'Hara, A., Kaiserli, E., Baumeister, R., Schafer, E., Nagy, F., Jenkins, G. I. \&Ulm, R. (2011). Perception of UV-B by the Arabidopsis UVR8 protein. Science 332(6025): 103-106.

Robson, P. R., McCormac, A. C., Irvine, A. S. \&Smith, H. (1996). Genetic engineering of harvest index in tobacco through overexpression of a phytochrome gene. Nat Biotechnol 14(8): 995-998.

Rockwell, N. C., Su, Y. S. \&Lagarias, J. C. (2006). Phytochrome structure and signaling mechanisms. Annu Rev Plant Biol 57: 837-858.

Ruckle, M. E., DeMarco, S. M. \&Larkin, R. M. (2007). Plastid signals remodel light signaling networks and are essential for efficient chloroplast biogenesis in Arabidopsis. Plant Cell 19(12): 3944-3960.

Runge, S., Sperling, U., Frick, G., Apel, K. \&Armstrong, G. A. (1996). Distinct roles for lightdependent NADPH:protochlorophyllide oxidoreductases (POR) A and B during greening in higher plants. Plant J 9(4): 513-523.

Sharrock, R. A. (2008). The phytochrome red/far-red photoreceptor superfamily. Genome Biol 9(8): 230.

Shen, H., Zhu, L., Castillon, A., Majee, M., Downie, B. \&Huq, E. (2008). Light-induced phosphorylation and degradation of the negative regulator PHYTOCHROMEINTERACTING FACTOR1 from Arabidopsis depend upon its direct physical interactions with photoactivated phytochromes. Plant Cell 20(6): 1586-1602.

Somers, D. E., Devlin, P. F. \&Kay, S. A. (1998). Phytochromes and cryptochromes in the entrainment of the Arabidopsis circadian clock. Science 282(5393): 1488-1490.

Srivastava, A., Lake, V., Nogaj, L. A., Mayer, S. M., Willows, R. D. \&Beale, S. I. (2005). The Chlamydomonas reinhardtii gtr gene encoding the tetrapyrrole biosynthetic enzyme glutamyl-trna reductase: structure of the gene and properties of the expressed enzyme. Plant Mol Biol 58(5): 643-658.

Staneloni, R. J., Rodriguez-Batiller, M. J., Legisa, D., Scarpin, M. R., Agalou, A., Cerdan, P. D., Meijer, A. H., Ouwerkerk, P. B. \&Casal, J. J. (2009). Bell-like homeodomain selectively regulates the high-irradiance response of phytochrome A. Proc Natl Acad Sci U S A 106(32): 13624-13629.

Stephenson, P. G., Fankhauser, C. \&Terry, M. J. (2009). PIF3 is a repressor of chloroplast development. Proc Natl Acad Sci U S A 106(18): 7654-7659.

Stephenson, P. G. \&Terry, M. J. (2008). Light signalling pathways regulating the Mgchelatase branchpoint of chlorophyll synthesis during de-etiolation in Arabidopsis thaliana. Photochem Photobiol Sci 7(10): 1243-1252.

Strasser, B., Sanchez-Lamas, M., Yanovsky, M. J., Casal, J. J. \&Cerdan, P. D. (2010). Arabidopsis thaliana life without phytochromes. Proc Natl Acad Sci U S A 107(10): 4776-4781.

Strayer, C., Oyama, T., Schultz, T. F., Raman, R., Somers, D. E., Mas, P., Panda, S., Kreps, J. A. \&Kay, S. A. (2000). Cloning of the Arabidopsis clock gene TOC1, an autoregulatory response regulator homolog. Science 289(5480): 768-771.

Takano, M., Inagaki, N., Xie, X., Kiyota, S., Baba-Kasai, A., Tanabata, T. \&Shinomura, T. (2009). Phytochromes are the sole photoreceptors for perceiving red/far-red light in rice. Proc Natl Acad Sci U S A 106(34): 14705-14710.

Tanaka, R. \&Tanaka, A. (2007). Tetrapyrrole biosynthesis in higher plants. Annu Rev Plant Biol 58: 321-346.

Toledo-Ortiz, G., Huq, E. \&Quail, P. H. (2003). The Arabidopsis basic/helix-loop-helix transcription factor family. Plant Cell 15(8): 1749-1770. 
Viczian, A., Kircher, S., Fejes, E., Millar, A. J., Schafer, E., Kozma-Bognar, L. \&Nagy, F. (2005). Functional characterization of phytochrome interacting factor 3 for the Arabidopsis thaliana circadian clockwork. Plant Cell Physiol 46(10): 1591-1602.

Wang, Y., Wu, J. F., Nakamichi, N., Sakakibara, H., Nam, H. G. \&Wu, S. H. (2011). LIGHTREGULATED WD1 and PSEUDO-RESPONSE REGULATOR9 form a positive feedback regulatory loop in the Arabidopsis circadian clock. Plant Cell 23(2): 486498.

Wang, Z. Y., Kenigsbuch, D., Sun, L., Harel, E., Ong, M. S. \&Tobin, E. M. (1997). A Mybrelated transcription factor is involved in the phytochrome regulation of an Arabidopsis Lhcb gene. Plant Cell 9(4): 491-507.

Wu, J. F., Wang, Y. \&Wu, S. H. (2008). Two new clock proteins, LWD1 and LWD2, regulate Arabidopsis photoperiodic flowering. Plant Physiol 148(2): 948-959.

Yamashino, T., Matsushika, A., Fujimori, T., Sato, S., Kato, T., Tabata, S. \&Mizuno, T. (2003). A Link between circadian-controlled bHLH factors and the APRR1/TOC1 quintet in Arabidopsis thaliana. Plant Cell Physiol 44(6): 619-629.

Yanovsky, M. J., Mazzella, M. A. \&Casal, J. J. (2000). A quadruple photoreceptor mutant still keeps track of time. Curr Biol 10(16): 1013-1015.

Yanovsky, M. J., Mazzella, M. A., Whitelam, G. C. \&Casal, J. J. (2001). Resetting of the circadian clock by phytochromes and cryptochromes in Arabidopsis. J Biol Rhythms 16(6): 523-530.

Yanovsky, M. J. Casal., J.J \& Whitelam, G.C. (1995). Phytochrome A, phytochrome B and HY4 are involved in hypocotyl growth responses to natural radiation in Arabidopsis: weak de-etiolation of the phyA mutant under dense canopies. Plant Cell \& Environment 18: 788-794.

Yu, J. W., Rubio, V., Lee, N. Y., Bai, S., Lee, S. Y., Kim, S. S., Liu, L., Zhang, Y., Irigoyen, M. L., Sullivan, J. A., Lee, I., Xie, Q., Paek, N. C. \&Deng, X. W. (2008). COP1 and ELF3 control circadian function and photoperiodic flowering by regulating GI stability. Mol Cell 32(5): 617-630.

Zeilinger, M. N., Farre, E. M., Taylor, S. R., Kay, S. A. \&Doyle, F. J., 3rd (2006). A novel computational model of the circadian clock in Arabidopsis that incorporates PRR7 and PRR9. Mol Syst Biol 2: 58.

Zhang, H., He, H., Wang, X., Yang, X., Li, L. \&Deng, X. W. (2011). Genome-wide mapping of the HY5-mediated gene networks in Arabidopsis that involve both transcriptional and post-transcriptional regulation. Plant J 65(3): 346-358.

Zhu, D., Maier, A., Lee, J. H., Laubinger, S., Saijo, Y., Wang, H., Qu, L. J., Hoecker, U. \&Deng, X. W. (2008). Biochemical characterization of Arabidopsis complexes containing CONSTITUTIVELY PHOTOMORPHOGENIC1 and SUPPRESSOR OF PHYA proteins in light control of plant development. Plant Cell 20(9): 2307-2323.

Zuo, Z., Liu, H., Liu, B., Liu, X. \&Lin, C. (2011). Blue-light dependent interaction of CRY2 with SPA1 regulates COP1 activity and floral initiation in Arabidopsis. Curr Biol 21(10): 841-847. 


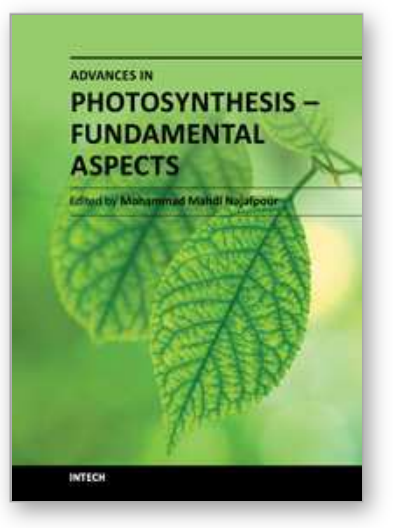

\section{Advances in Photosynthesis - Fundamental Aspects \\ Edited by Dr Mohammad Najafpour}

ISBN 978-953-307-928-8

Hard cover, 588 pages

Publisher InTech

Published online 15, February, 2012

Published in print edition February, 2012

Photosynthesis is one of the most important reactions on Earth. It is a scientific field that is the topic of many research groups. This book is aimed at providing the fundamental aspects of photosynthesis, and the results collected from different research groups. There are three sections in this book: light and photosynthesis, the path of carbon in photosynthesis, and special topics in photosynthesis. In each section important topics in the subject are discussed and (or) reviewed by experts in each book chapter.

\section{How to reference}

In order to correctly reference this scholarly work, feel free to copy and paste the following:

Sabrina Iñigo, Mariana R. Barber, Maximiliano Sánchez-Lamas, Francisco M. Iglesias and Pablo D. Cerdán (2012). The Photomorphogenic Signal: An Essential Component of Photoautotrophic Life, Advances in Photosynthesis - Fundamental Aspects, Dr Mohammad Najafpour (Ed.), ISBN: 978-953-307-928-8, InTech, Available from: http://www.intechopen.com/books/advances-in-photosynthesis-fundamental-aspects/thephotomorphogenic-signal-an-essential-component-of-photoautotrophic-life

\section{INTECH}

open science | open minds

\section{InTech Europe}

University Campus STeP Ri

Slavka Krautzeka 83/A

51000 Rijeka, Croatia

Phone: +385 (51) 770447

Fax: +385 (51) 686166

www.intechopen.com

\section{InTech China}

Unit 405, Office Block, Hotel Equatorial Shanghai

No.65, Yan An Road (West), Shanghai, 200040, China

中国上海市延安西路65号上海国际贵都大饭店办公楼 405 单元

Phone: +86-21-62489820

Fax: +86-21-62489821 
(C) 2012 The Author(s). Licensee IntechOpen. This is an open access article distributed under the terms of the Creative Commons Attribution 3.0 License, which permits unrestricted use, distribution, and reproduction in any medium, provided the original work is properly cited. 